\title{
Web Application on Information System Based on Cloud Computing and Scene Simulation
}

\author{
Zhang Jianjun \\ Beijing Special Vehicle Institute \\ Beijing, China \\ e-mail: zjj6629@sohu.com \\ He Mingli \\ Beijing Special Vehicle Institute \\ Beijing, China
}

\author{
Fan Xiquan \\ Beijing Special Vehicle Institute \\ Beijing, China \\ Wu Guohui \\ Department of Command and Control \\ Wuhan Ordnance N.C.O. Academy \\ Wuhan, China
}

\begin{abstract}
Nowadays, with the development of science and technology and the improvement of people's living standard, Internet cloud computing develops the smart city. A smart city cloud was constructed. Based on the cloud computing, a novel information system was designed with Web. The traditional information system only had one-way data collection function. It could not be used for analysis and executive. The novel system adopted closed-loop topology structure. Efficient sensor data protocol was discussed. $B / S$ service software was developed by MS ASP+SQL Server database. 3D interface was built by using TWaver MONO DESIGN development tool. The system can help us achieve the purpose of acquiring information from sensor and controlling actors based on Web service, optimizing users' experience compared to the traditional system.
\end{abstract}

Keywords- information system; cloud computing; monitoring control; sensor

\section{INTRODUCTION}

Smart city is the informationization development to a higher stage[1]. The rapid development of Internet technology boosts the construction of smart city, achieves more thorough perception, more extensive interconnection and more intelligent[2]. Cloud computing is a new service model and calculation mode based on Internet, with virtualization, scalability, multi-tenant characteristics and Web applications don't need to install specialized client software. they provide support means in order to solve the problems of large scale distributed data management, application integration oriented service, and the rapid deployment of resources.

\section{THE EXISTING MONITORING INFORMATION SYSTEM}

"Smart Plug" Wi-Fi intelligent electrical outlet (Fig.1) let your fingers touch every corner of the world[3]. 
network. The output of control strategy is produced as a monitoring result. Administrators query these strategies through the Html5 browser, and send confirmation instructions to the server. The server passes the instructions to the actuator. The running state of the equipment is adjusted by the actuator in the smart city cloud. At last, closed-loop control of environmental monitoring and protection is complete. Block diagram of closed-loop monitoring control system is shown as Fig.2.

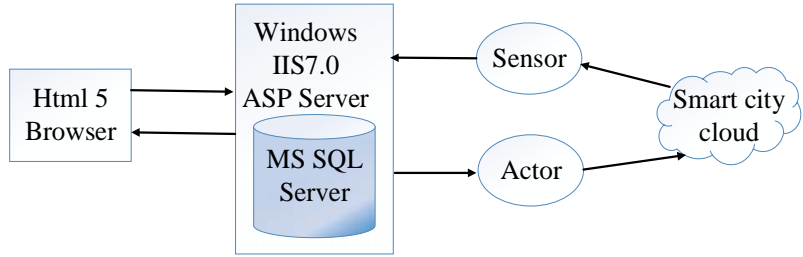

Figure 2. Block diagram of closed-loop monitoring information system.

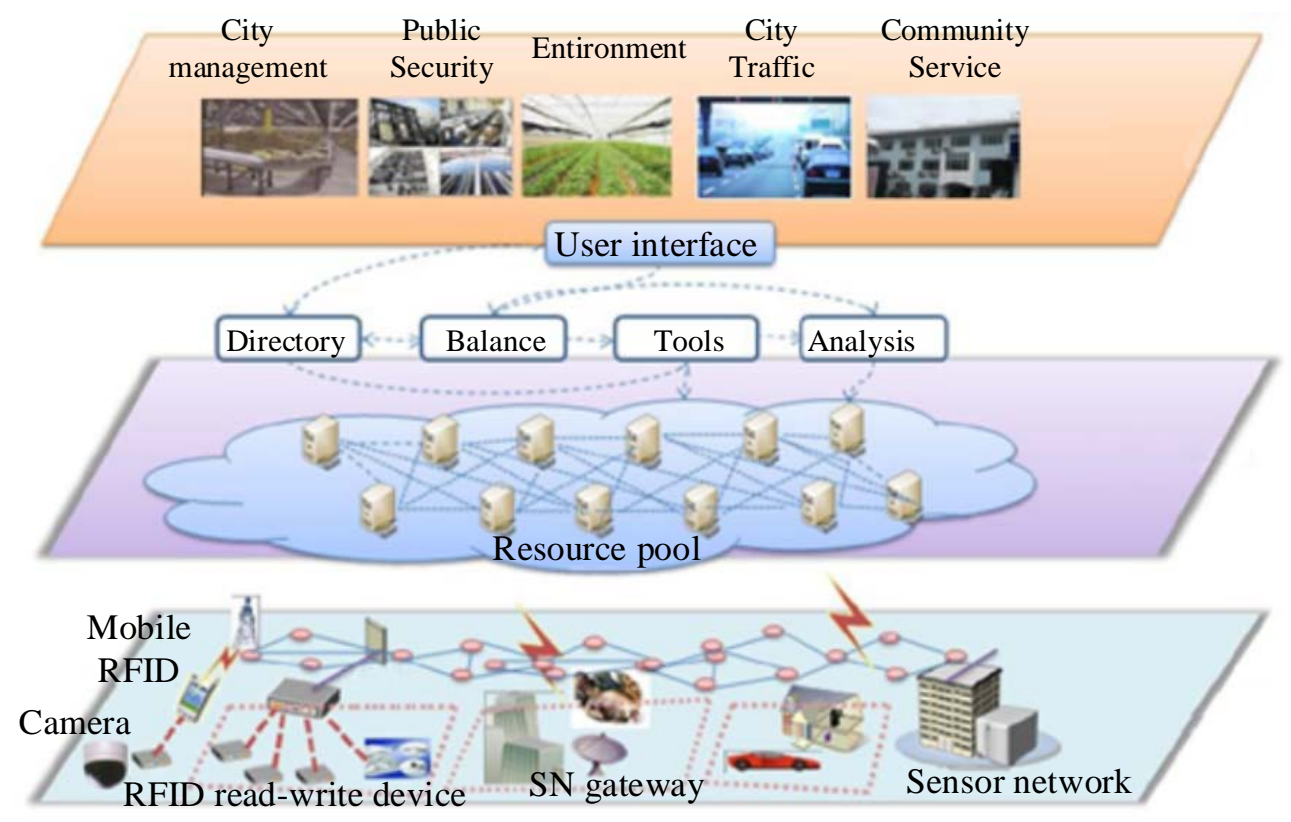

Figure 3. The system application mode.

In order to ensure a high degree of reliability, the system uses stable, good performance of the ASP + MS SQLServer2008 Architecture. At present, the market share of the architecture is about $50 \%$ in Chinese network area [4], and the technology is very mature. Maximum load capacity of the system can process thousands of people-level access. Under the premise of practical and reliable, the system uses the latest design concepts - Ajax, to achieve to update data without refreshing the whole webpage. The monitoring system application mode is shown as Fig.3.

\section{B. Design of monitoring data communication protocol}

1) The overall monitoring data protocol framework. All communication protocol follows the protocol framework. A data frame structure is given as follow:
TABLE I. A DATA FRAME STRUCTURE

\begin{tabular}{|l|c|}
\hline \multicolumn{1}{|c|}{ Item } & Defined length \\
\hline head & 2B \\
\hline MAC & $6 \mathrm{~B}$ \\
\hline type & $1 \mathrm{~B}$ \\
\hline control & $1 \mathrm{~B}$ \\
\hline destination & $2 \mathrm{~B}$ \\
\hline source & $2 \mathrm{~B}$ \\
\hline Length & $1 \mathrm{~B}$ \\
\hline Domain & $1 \mathrm{~B} 240 \mathrm{~B}$ \\
\hline CRC & $2 \mathrm{~B}$ \\
\hline tail & $2 \mathrm{~B}$ \\
\hline
\end{tabular}

- Head: frame start delimiter, defined as two bytes 0xAA BB.

- MAC address: MAC address of Wi-Fi module in a relay device, 6 bytes, used to distinguish between users.

- Data type: uplink data for 0x01; downlink data for 0x02.

- Control word: 0x01: normal mode data information; 0x02: normal mode configuration information; 0x03: 
the code model configuration information; 0x04: advanced configuration information; 0x05: the heart communication; 0x06: server special protocol.

- Destination address: the server address can be fixed for a specific address.

- Data length: the length of data streams.

- Data domain: control word content.

- $\quad$ CRC check: CRC16 check part, integrity checking of data packet.

- Tail: end of frame delimiter, defined as two bytes 0x55 FF.

- A node address is randomly generated from 0x00 00 to $0 \mathrm{xFE} \mathrm{FF}, 0 \mathrm{xFF} 00$ to $0 \mathrm{xFF}$ FF for the proper address cannot be assigned to the nodes. For example: the server address is 0xFF 00, the client address is $0 x F F 10$, the code model node address is 0xFF 20, the code model relay device address is 0xFF 30, the broadcast address is 0xFF FF.

2) Analysis of configuration information communication protocol under normal mode.

- Destination address: query address information for all nodes is 0xFF FF.

- Data domain: 0xFF.

The data doman content is given as follow:
TABLE II. THE DATA DOMAIN CONTENT

\begin{tabular}{|l|c|}
\hline \multicolumn{1}{|c|}{ Item } & Defined length \\
\hline type & $2 \mathrm{~B}$ \\
\hline address & $6 \mathrm{~B}$ \\
\hline number & $1 \mathrm{~B}$ \\
\hline sensor/actor address & $1 \mathrm{~B}$ \\
\hline destination & $2 \mathrm{~B}$ \\
\hline actor status & $2 \mathrm{~B}$ \\
\hline value & $1 \mathrm{~B}$ \\
\hline time & $1 \mathrm{~B} \sim 240 \mathrm{~B}$ \\
\hline
\end{tabular}

\section{The Web 3D monitoring control interface}

Web 3D is a kind of virtual reality technology and interactive browsing operation which presents threedimensional devices of the smart city by Internet platform. The tool we use is the newest flagship product TWaver MONO DESIGN developed by Serva Corporation. Built on techniques including HTML5, WebGL and JavaScript. It provides us a complete web $3 \mathrm{D}$ rendering engine, a visualized 3D modeling editor, a template library at the industry level and API interfaces for programming [5]. TWaver HTML5 package has the complete functions including demo applications with source code, developer guide and API document. We establish complete Web-based general components like GUI, professional network topology, equipment pane, tree and table. The monitoring control system supports Mainstream browsers like Chrome, Firefox, Safari, IE9+, Opera and etc. The Environmental monitoring control scene is shown as Fig.4.

The results of monitoring are shown as Fig.5 and Fig.6.

As can be seen from Fig.5 and Fig.6, the results are used to optimize the air conditioning system in the smart city cloud by controlling actors, enables it to achieve the optimal state, reducing the carbon footprint index maximum.

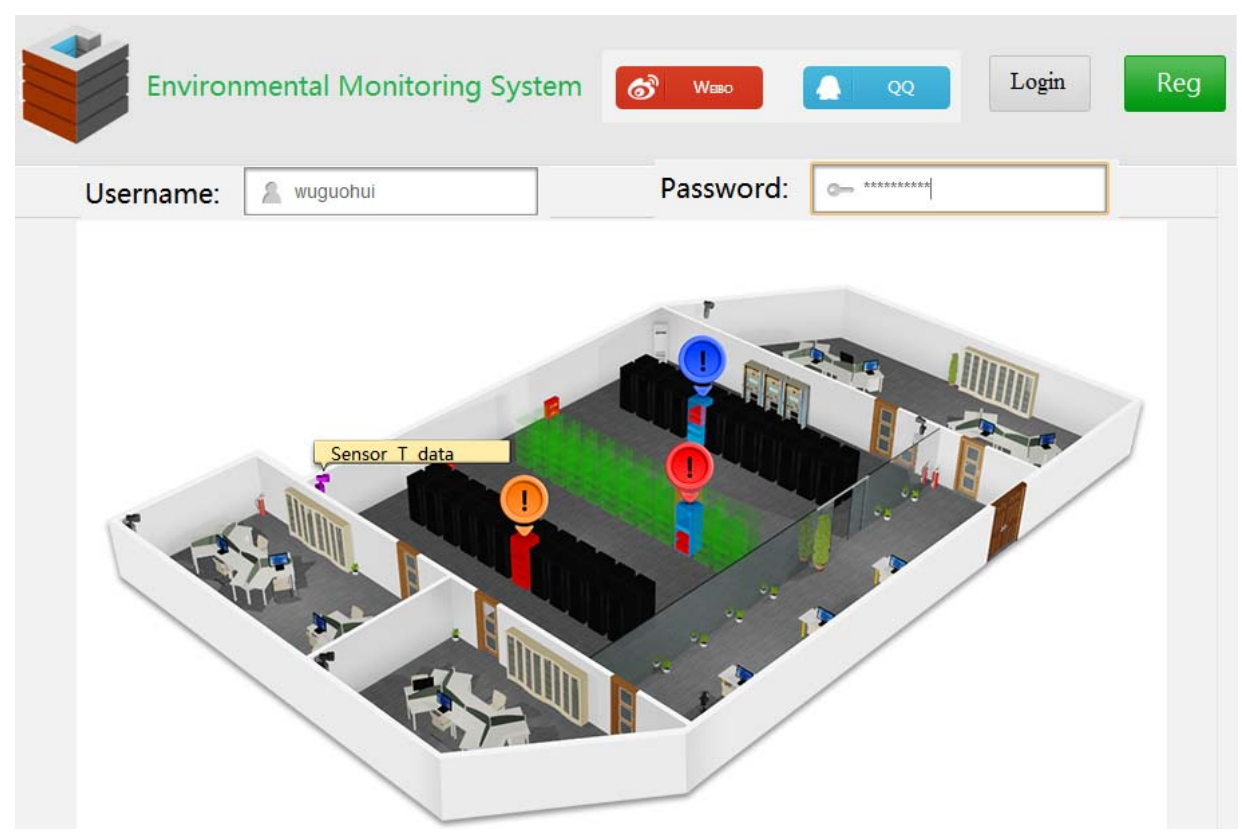

Figure 4. The Environmental monitoring control scene on Web 3D. 


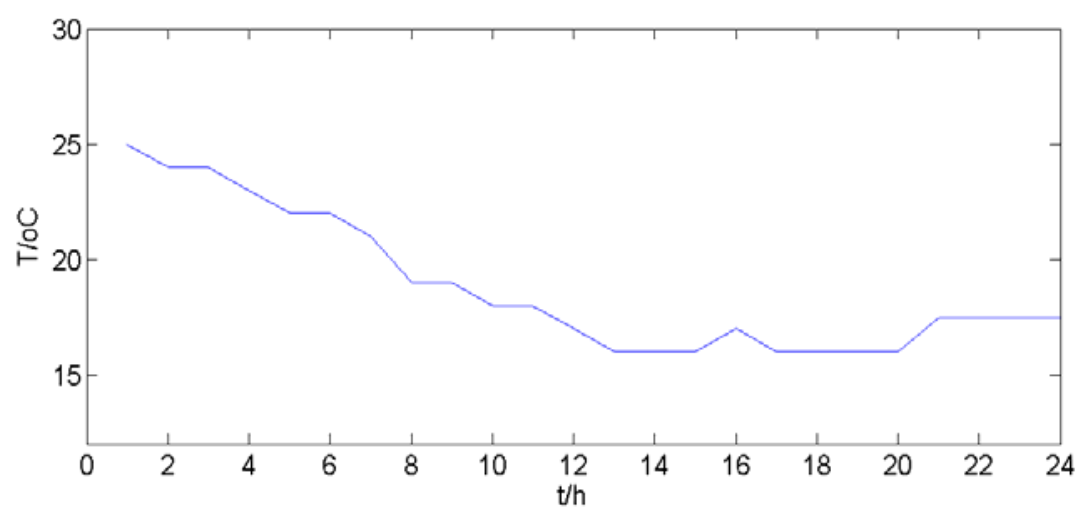

Figure 5. Temperature variation curve.

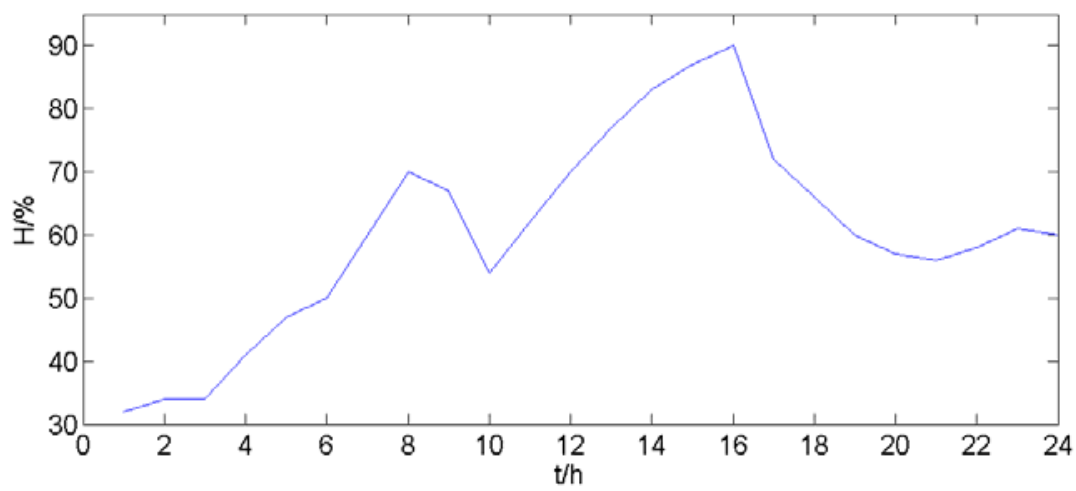

Figure 6. Humidity variation curve.

\section{CONCLUSIONS}

Cloud computing will greatly promote the economic transformation and sustainable development. Information system will enter a new mode. Making use of 3D scene simulation based on Web service will be better users' experience. Through technological innovation, based on cloud computing to construct and perfect the closed-loop monitoring control system is the route one must take to enhance information-based level.

\section{REFERENCES}

[1] Huang Xin-guang, Wei Jin-wu, Liu lu, Zhang Yun-yong, Smart City Construction and Development Research. Telecommunications Network Technology, 2011(9),pp.27-35,2011.

[2] Shen Ming, Zhou Qi-gang, Shao Wen-yan, The foundation for the application of smart city based on the information integrated platform constructed by Cloud Computing, Communications World Weekly, pp. 38-39,2012.

[3] Ma Yan, The Design and Application of Intelligent Electrical Outlet for Campus's Electricity Saving and Emission Reduction. Bulletin of Science and Technology, 29(9), pp. 102-105,2013.

[4] Wu Guo-hui, He Le, Wang Xiao-wei, Wei Kan, Study on Integrated Method of Files Management Based on Cooperative OA. 2010 1st International Conference on Internet technology and application process, 2010.

[5] Twaver-Network Toplogy Software, http://twaver.servasoft.com, 2014. 